\title{
Characterization of Headache in COVID-19: a Retrospective Multicenter Study
}

\author{
Rafael César dos Anjos de Paula ${ }^{1}$ Thaís de Maria Frota Vasconcelos ${ }^{1}$. Francisco Bruno Santana da Costa ${ }^{1}$. \\ Lara Albuquerque de Brito $^{2}$ - Danielle Mesquita Torres ${ }^{2}$. Alissa Elen Formiga Moura ${ }^{2}$. Danilo Nunes Oliveira ${ }^{1}$. \\ Guilherme Alves de Lima Henn ${ }^{3,4}$. Pedro Gustavo Barros Rodrigues ${ }^{1} \cdot$ Isabelle de Sousa Pereira ${ }^{1}$. \\ lanna Lacerda Sampaio Braga ${ }^{5}$. Felipe Araújo Rocha ${ }^{2}$. Norberto Anízio Ferreira Frota ${ }^{2,6}$. \\ Fernanda Martins Maia Carvalho ${ }^{2,6}$. Milena Sales Pitombeira ${ }^{2}$. José Wagner Leonel Tavares-Junior ${ }^{1}$. \\ Raquel Carvalho Montenegro ${ }^{7}$ Pedro Braga-Neto ${ }^{1,8} \cdot$ Paulo Ribeiro Nóbrega ${ }^{1,2} \oplus$ - Manoel Alves Sobreira-Neto ${ }^{1,9}$
}

Received: 5 January 2021 / Accepted: 12 May 2021 / Published online: 25 May 2021

(c) The Author(s), under exclusive licence to Springer Science+Business Media, LLC, part of Springer Nature 2021

\begin{abstract}
Headache is the most common neurological symptom in COVID-19, reported in 6.5 to $34 \%$ of patients. Few studies have analyzed its characteristics, and some of them included cases without laboratory confirmation or reported only critical patients. We aimed to analyze the clinical characteristics of COVID-19 associated headache in laboratory-confirmed cases. We conducted a retrospective evaluation of patients with COVID-19 and neurological symptoms. Patients who reported headache answered an interview about its clinical characteristics. Twenty-four patients with COVID-19 associated headache completed the interview. Mean age of patients was 53.8 (standard deviation-17.44), and 14 out of 24 (58.3\%) were male. The majority (75\%) had no previous history of headache. Fever was documented in 19 out of the 24 patients (79.1\%). Headache was predominantly bifrontal or holocranial, in pressure, during hours, worsening with cough or physical activity. COVID19 headache tends to appear in the first days of symptoms, be either frontal or holocranial and last for days. The quality of pain in pressure and the worsening with cough or physical activity were reported in most cases. We have not found any characteristic that could differentiate COVID-19 associated headache from other causes of headache, possibly because of its multifactorial mechanism.
\end{abstract}

Keywords Headache $\cdot$ Neurological symptoms $\cdot$ Coronavirus $\cdot$ COVID-19 $\cdot$ Characterization

\section{Introduction}

In mid-November 2019, an outbreak of pneumonia of unknown cause in the city of Wuhan, Hubei province, China, began to attract the attention of Chinese health authorities [1]. The virus was identified as a new type of coronavirus

Paulo Ribeiro Nóbrega

paulo_r_med@yahoo.com

1 Division of Neurology, Department of Clinical Medicine, Universidade Federal Do Ceará, Fortaleza, Brazil

2 Department of Neurology, Hospital Geral de Fortaleza, Fortaleza, Brazil

3 Division of Infectious Diseases, Department of Clinical Medicine, Universidade Federal Do Ceará, Fortaleza, Brazil

4 Department of Infectious Diseases, Hospital São José de Doenças Infecciosas, Fortaleza, Brazil
(CoV), dubbed SARS-CoV-2, and its associated disease received the name of COVID-19 [1]. One year later, more than forty-nine million cases around the world had already been confirmed with SARS-CoV-2, and over one million and two hundred fifty thousand people died. In Brazil, more than five million and six hundred thousand were infected,

5 Department of Gerontology, Hospital Geral Cesar Cals, Fortaleza, Brazil

6 Universidade de Fortaleza, Fortaleza, Brazil

7 Núcleo de Pesquisa E Desenvolvimento de Medicamentos, Universidade Federal Do Ceará, Fortaleza, Brazil

8 Center of Health Sciences, Universidade Estadual Do Ceará, Fortaleza, Brazil

9 Centro Universitário Christus, Fortaleza, Brazil 
and more than one hundred and sixty thousand have died because of this disease [2].

COVID-19 was primarily characterized as a respiratory disease; however, with the increase in the number of cases, reports emerged stating that the disease affected other organs and systems, with some studies reporting a prevalence above $35 \%$ of patients with neurological symptoms [3]. The main theory is that the SARS coronavirus 2 probably uses the olfactory nerves as the primary entry port into the central nervous system (CNS), with a high viral load allowing the interaction of the viral spike (S) protein with the ACE2 receptor expressed in the endothelium [4].

Headache is the most common neurological symptom in COVID-19, reaching a prevalence between 6.5 and $34 \%$ [5-7]. It is usually accompanied by fever [8] and may present as the only symptom of the infection [9]. Many studies about COVID-19 associated headache were conducted on seriously ill patients, were reported in single cases or small case series, included a majority of patients with an unconfirmed clinical diagnosis, or had headache as a secondary outcome measured $[3,10,11]$. In the present study, we aimed to evaluate all consecutive patients with a laboratory-confirmed diagnosis of COVID-19 with neurological symptoms for the prevalence of headache and describe its characteristics.

\section{Material and Methods}

This was an observational and retrospective study. Patients over 18 years of age diagnosed with COVID-19 who had neurological symptoms were evaluated for the presence of headache and its characteristics.

Clinical charts of patients with a recent diagnosis of COVID-19, confirmed by PCR or serology, were evaluated by the researchers for the presence of neurological symptoms, such as altered level of consciousness, focal neurological deficits, anosmia, ageusia, epileptic seizures, or persistent headache. The study was carried out in the clinical wards and intensive care units (ICUs) of the following hospitals: Hospital Universitário Walter Cantído (HUWC), Hospital Geral de Fortaleza (HGF), and Hospital São José de Doenças Infecciosas (HSJ). Demographic characteristics, comorbidities, severity of clinical presentation, laboratory test results, and headache characteristics were described. We screened all consecutive cases from May 22 to July 20, 2020.

The diagnosis of headache was not restricted to the International Classification of Headache Disorders, 3rd version (ICHD-3) criteria for specific secondary headaches [12]. Every patient that complained about headache during the course of the disease was included.

In patients with clinical symptoms, diagnosis of COVID19 was confirmed by real-time reverse transcriptase polymerase chain reaction (RT-PCR) assay (Allplex ${ }^{\mathrm{TM}}$ SARS-CoV-2 Assay (N/RdRP/S genes), Seegene Brazil and Kit Molecular SARS-CoV-2 (E/RP), Bio-Manguinhos/ Fiocruz, Brazil) from oropharyngeal-nasopharyngeal swab, sputum, or lower respiratory tract sample or by the presence of anti-SARS-CoV-2 IgM antibodies (LIAISON ${ }^{\circledR}$ SARS-CoV-2 IgM; LIAISON® SARS-CoV-2 S1/S2 IgG, DiaSorin, Saluggia, Italy).

\section{Variables}

We assessed demographic variables, including age, sex, prior history of hypertension, diabetes, chronic renal disease, obesity, smoking habit (current or in the previous 6 months), cardiovascular diseases, chronic pulmonary diseases, sleep obstructive apnea, cancer, and immunocompromised conditions, including HIV. We reported the use of angiotensinconverting enzyme inhibitors (ACEi), angiotensin II receptor blockers (ATII), systemic steroids, and other medications.

Clinical evaluation consisted of anamnesis and general physical and neurological examination. We analyzed the presence of prior history of headache and its characteristics. Neurological examination was performed following a standard form. For the assessment of pain intensity, an analog visual scale (VAS) was used.

Characteristics of headache associated with COVID-19 were described, including time of onset; severity; location; pain quality; duration; response to analgesics; associated symptoms (e.g., nausea, vomiting); and factors of worsening, such as physical activity or cough.

The laboratory tests done were defined by the attending physicians, and their results were collected, which included complete blood count, electrolytes, bilirubins, D-dimer, thyroid hormones, fasting blood glucose, lactate, and arterial blood gases.

\section{Ethical Aspects}

This study complies with the ethical principles of the Declaration of Helsinki. Patients who agreed to participate in the study signed an informed consent form after a researcher explained the aim of the study, its duration, and the implications of participation.

The study was approved by the research ethics committee of HUWC under registration number 4092933.

\section{Statistical Analysis}

This was a descriptive study. Categorical variables were described as absolute frequencies and percentages, while numerical variables were described as averages and standard deviations. Comparative analyses between patients with and without headache were done using Chi-square test for 
categorical variables. Two-tailed $p$ values of $<0.05$ were considered statistically significant. Statistical analyses were performed with IBM-SPSS version 18.0 (IBM, Armonk, NY, USA).

\section{Results}

During the study period, May 22 to July 20, 2020, we have identified 108 patients with laboratory-confirmed COVID19 infection and neurological symptoms. The interview was completed with 101 patients, who composed our final sample. Headache was reported by 28 patients $(27.7 \%)$ being the second most prevalent neurological symptom (Table 1). Twenty-four patients accurately responded all headache related questions.

Patients with COVID-19-associated headache were mostly male (14 out of $24-58.3 \%$ ) with a mean age of 53.8 (17.44) (Table 2). Presence of any of the evaluated comorbidities was reported in 18 out of 24 patients (75\%). Hypertension (41.6\%) and diabetes $(16.6 \%)$ were the most common comorbidities. Three patients had a previous neurological condition, namely, one had a previous stroke, another one had epilepsy, and another had chronic vestibular vertigo. Need for supplementary oxygen was reported by half of all patients (12 of 24). Only one patient needed mechanical ventilation.

Patients with headache were more likely to have fever than those without ( 19 of 24 vs 24 of 56, p = 0.0028). Fever in any moment during the disease period was documented in 19 of the 24 patients (79.1\%), which occurred mainly in a different moment from the headache. Fever concomitant with headache was identified in only two patients $(8.3 \%)$.

Among patients who presented headaches during COVID-19 infection, most of them (50\%) occurred in the first day of symptoms. One of the 24 patients presented headache on the tenth day, which was the maximum interval

Table 1 Distribution of neurological symptoms in 101 patients with COVID-19 and neurological symptoms $N(\%)$

\begin{tabular}{ll}
\hline Focal neurological deficit & $41(40.5 \%)$ \\
Headache & $28(27.7 \%)$ \\
Altered level of consciousness & $19(18.8 \%)$ \\
Hipo/anosmia & $17(16.8 \%)$ \\
Hipo/ageusia & $17(16.8 \%)$ \\
Seizures & $13(12.9 \%)$ \\
Myalgia & $12(11.9 \%)$ \\
Myoclonus & $4(3.9 \%)$ \\
Back pain & $4(3.9 \%)$ \\
Peripheral neuropathy & $2(1.9 \%)$ \\
Ataxia & $1(0.9 \%)$ \\
Others & $5(4.9 \%)$ \\
\hline
\end{tabular}

Table 2 Demographic variables, comorbidities, and laboratory confirmation methods in 24 patients with COVID-19-associated headache

\begin{tabular}{ll}
\hline Age, mean (SD) & $53.8(17.44)$ \\
Sex (males) $N(\%)$ & $14(58.3 \%)$ \\
Comorbidities & $18(75 \%)$ \\
Arterial hypertension & $9(37.5 \%)$ \\
Diabetes mellitus & $4(16.6 \%)$ \\
Obesity & $3(12.5 \%)$ \\
Chronic renal injury & $2(8.3 \%)$ \\
Chronic pulmonary disease & $2(8.3 \%)$ \\
Cancer & $2(8.3 \%)$ \\
Obstructive sleep apnea syndrome & $1(4.1 \%)$ \\
Tabagism & $4(16.6 \%)$ \\
Etilism & $3(12.5 \%)$ \\
Use of oxygen & $12(50 \%)$ \\
Mechanical ventilation & $1(4.1 \%)$ \\
Laboratory-confirmed diagnosis & $24(100 \%)$ \\
RT-PCR & $7(29.1 \%)$ \\
Serology (IgM) & $17(73.9 \%)$ \\
\hline
\end{tabular}

for the onset of headache in our sample (1-10 days). Headache persisted after other symptoms of viral infection had subsided in 3 patients (12.5\%).

The vast majority of patients (75\%) had no previous history of headache, and only $6(25 \%)$ already had a previous history of headache, of these 4 (66.7\%) reported having a change in headache pattern. The quantification of pain of COVID-19-related headache, using the VAS, had an average score of 6 (1.83). Most patients (75\%) had VAS scores of 6 or less, classifying pain as mild to moderate in intensity. When asked if the pain was disabling, being strong enough to impact the performance of basic activities of daily living (BADLs), most patients (70.8\%) said it was not disabling.

The majority of patients (95.8\%) took pain relief medications, including analgesics (dipyrone or acetaminophen) and NSAIDs (naproxen and ibuprofen), and most of them reported improvement in pain intensity after medication. The period of the day when pain was more prevalent was also asked, with 11 (45.8\%) patients reporting the highest prevalence in the morning, 7 (29.1\%) in the afternoon, and two $(8.3 \%)$ at night, while four (16.6\%) patients did not report a period of the day of more prevalence.

Headache duration was more than $1 \mathrm{~h}$ to less than $24 \mathrm{~h}$ in 15 patients $(62.5 \%)$, more than $24 \mathrm{~h}$ in $8(33.3 \%)$, and a couple of minutes in one patient (4.2\%). Regarding lateralization, 22 patients $(91.7 \%)$ had bilateral headache, 2 (8.3\%) reported a fixed unilateral headache, $1(4.1 \%)$ reported pain on the left side, and one $(4.1 \%)$ on the right side of the head (Table 3).

Headache location was predominantly frontal in 12 patients $(50 \%)$, holocranial in $8(33.3 \%)$, retroorbital in 2 
Table 3 Headache characteristics in 24 patients with COVID-19associated headache

\begin{tabular}{ll}
\hline Duration & \\
More than 1 day & $8(33.3 \%)$ \\
1-24 h & $15(62.5 \%)$ \\
Less than $1 \mathrm{~h}$ & $1(4.2 \%)$ \\
Lateralization & \\
Bilateral & $22(91.7 \%)$ \\
Unilateral & $2(8.2 \%)$ \\
Location & \\
Frontal & $12(50 \%)$ \\
Holocranial & $8(33.3 \%)$ \\
Retroorbital & $2(8.3 \%)$ \\
Temporal & $1(4.2 \%)$ \\
Occipital & $1(4.2 \%)$ \\
Pain quality & \\
Pressure & $12(50 \%)$ \\
Tightening & $9(37.5 \%)$ \\
Throbbing & $3(12.5 \%)$ \\
Associated symptoms & $14(58.3 \%)$ \\
Irritability & $4(16.6 \%)$ \\
Vertigo & $2(8.3 \%)$ \\
Pericranial allodynia & $2(8.3 \%)$ \\
Nausea & $2(8.3 \%)$ \\
Impaired concentration & $2(8.3 \%)$ \\
Vomiting & $1(4.1 \%)$ \\
Photophobia & $1(4.1 \%)$ \\
Adynamia & $1(4.1 \%)$ \\
Headache intensity & $17(70.8 \%)$ \\
VAS score of 6 or less (mild/moderate) \\
Impact in BADLs & \\
\hline
\end{tabular}

$V A S$ visual assessment scale, $B A D L s$ basic activities of daily living

(8.3\%), temporal in one (4.2\%), and occipital in one patient (4.2\%). Pain quality was characterized it as pressure in 12 patients (50\%), tightening in $9(37.5 \%)$, and throbbing in 3 patients $(12.5 \%)$.

Most patients (58.3\%) had associated symptoms, including irritability in 4 (16.6\%); nausea, vertigo, pericranial allodynia, and impaired concentration in 2 patients each (8.3\%); and vomiting, photophobia, and adynamia in 1 patient $(4.1 \%)$. The majority of patients $(70.8 \%)$ reported worsening of headache with routine physical activity or cough (Table 3).

Headache was mild to moderate in most cases, frontal or holocranial with pressure or tightening quality and without nausea, vomiting, phonophobia, or photophobia. These characteristics could lead to a phenotypic classification as tension-type headache according to ICHD-3 classification in the majority of patients, but the presence of fever and systemic signs of infection in most patients and a concomitant viral infection in all cases lead to an actual classification as "headache attributed to systemic viral infection."

Anosmia was present in $33 \%$ of the patients and was the initial symptom in four of these patients.

\section{Discussion}

We have analyzed all consecutive patients with COVID-19 infections and neurological symptoms for the presence of headache and described its characteristics. All of our patients had laboratory-confirmed recent COVID-19 infection (either by PCR or IgM, IgA serology), which was different from most previous studies where the majority of patients had a clinical diagnosis without laboratory confirmation [11]. Among 108 patients with confirmed COVID-19 and neurological symptoms, 28 had headache $(27.7 \%)$ being the second most common neurological symptom after focal neurological deficit (40.5\%), which is contrary from previous literature findings where headache was the most common neurological symptom $(6.5-34 \%)[3,5,13]$. An explanation for the high prevalence of focal neurological deficits may be the fact that among health centers in the study, there is a local reference center for cerebrovascular diseases with the largest stroke unit in the country, resulting in a considerable number of patients with stroke and concomitant COVID-19 infection being included.

Fever was present in most patients with headache (79.1\%), and it was more common in patients with headache than in those without. Headache can be associated with acute fever, although in most cases of COVID19 in our series (89.4\%) fever and headache were not concomitant, and some patients also had headache that lasted longer than fever and all other symptoms of acute viral illness, including cough, suggesting that headache was not a direct consequence of acute fever, as might be the case in other acute febrile diseases, but might be related to systemic infection, among other factors. The mechanisms causing headache in systemic disease include direct effects of the microorganisms themselves and effect of cytokines and other inflammatory mediators. Several cells are likely to be involved (activated microglia and monocytic macrophages, activated astrocytes, and blood-brain barrier and endothelial cells), along with several immunoinflammatory mediators (cytokines, glutamate, COX-2/PGE2 system, NO-iNOS system, and reactive oxygen species system) [12].

Most patients (50\%) had headache in the first day of symptoms, a fact that could raise awareness for patients presenting with new onset headache with or without other symptoms as a possible initial symptom of COVID-19 infection. Headache has been described as the sole presenting symptom of COVID in literature [9]. In our study, patients 
presented headache from the first up to the tenth day of symptoms, even after resolution of fever, which could suggest multiple mechanisms for headache depending on the moment it appears in the course of the disease.

Most patients in our study with COVID-19 associated headache did not have a previous history of headache, but $66 \%$ of those who had reported a change in headache pattern as found in literature $[9,13]$. During the ongoing COVID-19 pandemic, a new onset headache or a change in a previous pattern of headache should raise the suspicion of a new onset COVID-19 infection.

Headache lasted for an average of 6 days and a maximum of 10 days, consistent with literature on the subject with reports of headache lasting up to 14 days [9]. We have found headache to be predominantly bifrontal or holocranial, mostly with a pressure quality and duration of hours, consistent with other studies $[9,14,15]$. As for pain intensity, it was mild to moderate in most patients, and it did not impact basic activities of daily living (BALDs), differently from other reports and series, in which pain was mostly moderate to severe, impacting BALDs $[9,14,15]$. We have also found greater prevalence of pain in the morning and worsening of pain with cough or physical activity in most patients.

Although it is not completely clear how SARS-CoV 2 affects the nervous system, models and theories try to explain how this occurs. The main theory is that the SARS coronavirus 2 probably uses the olfactory nerves as a primery input port on the CNS through interaction of the viral S protein with the ACE2 receptor expressed on endothelium [4]. SARS-CoV 2 binds to the ACE2 receptor in the host cell, releasing virion particles. The virus RNA gets attached to the host's ribosomes producing polyproteins that will be processed by proteolytic enzymes and result in new virions, which promotes the spread of infection [16]. After viral replication and cell damage, there is a dysregulation of ACE2 receptors (downregulation), with modulation of the host's immune system to a highly inflammatory state. SARS-CoV 2, when binding to the ACE2 receptor, causes accumulation of angiotensin II (Ang II), which has several potentially deleterious effects, among them a proinflammatory effect [17].

The response to inflammation in the brain appears to be centered around microglia. In a resting state, ramified microglia (M0) are constantly scanning the neuropil through their highly motile processes. After encountering an insult signal microglia change to an ameboid activated state and release cytokines. Short or moderate signals lead to a neuroprotective M2 phenotype, whereas intensive acute (such as a neurotropic viral infection) or chronic activation renders a proinflammatory $\mathrm{M} 1$ phenotype producing reactive oxygen species; nitric oxide; proteases; and proinflammatory cytokines such as IL-1 $\beta$, IL- 6 , and tumor necrosis factor- $\alpha$ (TNF- $\alpha$ ), all of which may endanger neuronal populations [18]. Microglia also activates astrocytes that can modulate the recruitment and activation of additional microglial and other immunocompetent cells to the injury site closing the circle of a reactive positive feedback potentiating brain inflammation [19].

The viral load of Sars-Cov-2 detected from patient respiratory tracts has been positively linked to lung disease severity and to plasma angiotensin II levels [20]. Detection of viral RNA in plasma and blood viral load have also been correlated to systemic disease severity [21]. Coronavirus replication in pulmonary alveoli might result in breakdown of alveolar vessels and virus leakage into the blood flow, through which the virus is disseminated across the whole body [22] and can enter different organs, including the CNS, through interaction with ACE2 receptors. A higher viral load could also potentiate direct CNS invasion from the upper airways through olfactory nerves expressing ACE2 receptors, but we have found no evidence connecting increased viral loads to presence or severity of neurological symptoms.

In the human brain, ACE2 expression is in vascular distribution and in neurons of the motor cortex, lentiform nucleus, thalamus, rafe nuclei, solitary tract, and ambiguous nucleus [23]. Deregulation of Ang II/ACE2/angiotensin 1-7 has been reported in stroke, cognitive decline, Alzheimer's disease, and Parkinson's disease in addition to headache [17, 23]. The production of Ang II locally in the neurons of rat, human dorsal root ganglia (DRG), and its co-localization with substance $\mathrm{P}$ and calcitonin gene-related peptide (CGRP) may indicate a participation of Ang II in the regulation of nociception [24].

There is no consensus on how SARS-CoV 2 affects the nervous system, and the mechanisms involved in COVID-19 headache are also unclear. The first possibility is based on the direct invasion at trigeminal nerve endings in the nasal cavity by SARS-CoV 2 [13]. Experimental and clinical animal studies report the potential spread via retrograde axonal transport from peripheral nerves such as the olfactory nerve [25]. Nevertheless, our study found that only $33 \%$ of patients with headache had anosmia at some point in the course of the disease, which is less than previous reports [26]. This fact contradicts the theory of the invasion via the olfactory nerves being associated with COVID-19 headache. The possibility of a similar invasion through trigeminal, instead of olfactory nerve endings, still remains. However, the presence of the ACE2 receptor would be a crucial component for viral binding, and although its presence has been demonstrated in several locations in the brain, ACE2 expression in trigeminal terminations has not yet been demonstrated [17].

A second possible mechanism for COVID-19 headache could be a large release of cytokines and inflammatory mediators such as interleukin 1 beta, nitric oxide, nuclear factor kappa B, and prostaglandin E2 activating the peripheral trigeminal endings [17]. A so-called cytokine storm can occur in COVID-19 patients involving the massive release 
of inflammatory mediators including cytokines, glutamate, $\mathrm{PGE}_{2}, \mathrm{NO}$, and reactive oxygen species which affect the hypothalamus triggering fever, along with activation of microglia, macrophages, astrocytes, and endothelial cells, disrupting the blood brain barrier [27]. This same release of cytokines can lead to widespread pulmonary involvement and multiorgan failure in some patients [28].

Neurogenic inflammation refers to inflammation that results from activation of sensory nerve fibers [29]. At the peripheral ending of these fibers, substance $P$, calcitonin gene-related peptide (CGRP), and neurokinin A (NKA) are released which leads to activation of endothelial cells, mast cells, macrophages, $\mathrm{T}$ cells, and dendritic cells. These cells release numerous other substances, such as prostaglandins, tumor necrosis factor alpha (TNF $\alpha$ ), interleukins, glutamate, nerve-growth factor (NGF), and vasoactive intestinal peptide (VIP) also causing plasma protein extravasation and blood-brain barrier dysfunction [30], which helps to maintain an inflammatory state in nervous tissues. The process of neurogenic inflammation can be self-amplifying, where glutamate, the major excitatory neurotransmitter, plays a key role in sensitization of sensory nerve fibers and also long-term potentiation through CNS pain centers' sensitization [30]. This process has been well documented mostly in migraine [31], where it serves as a therapeutic target for monoclonal antibodies directed to CGRP or CGRP receptors, but also in other primary headaches [32]. It may also play a role in COVID-19-associated headache.

A third possibility is vascular pathogenesis caused by endothelial dysfunction (richly expressing ACE2) as well as diffuse endothelial inflammation, which might have an important role in trigeminal activation leading then to headache $[17,23]$. Data shows that SARS-CoV 2 triggers a cytokine cascade exacerbating ischemic brain damage in a similar way as influenza virus. These similarities might suggest that cerebrovascular endothelial dysfunction mediated by cytokine release may be one possible mechanism leading to cerebrovascular disease and headache [27, 33]. Endothelial dysfunction associated with a pro-thrombotic state could be one of the reasons of the increased incidence of stroke in COVID-19 patients with severe illness of 0.5 to $3 \%$ [34, 35].

In patients with severe and persistent headache associated with COVID19 who underwent cerebrospinal fluid (CSF) analysis in a recently published case series, $11 / 13(84.6 \%)$ had intracranial hypertension (CSF opening pressure $>200 \mathrm{mmH}_{2} \mathrm{O}$ ) in the absence of meningitis/ encephalitis, while $6 / 13$ (46.1\%) had CSF opening pressure $>250 \mathrm{mmH}_{2} \mathrm{O}$ and 2 had papilledema, suggesting a role for intracranial hypertension in some cases of COVID19 associated headache [36]. As our study was observational, we did not require the collection of CSF, which makes it impossible to investigate a possible role of CSF hypertension, as well as viral meningitis or encephalitis, in headache mechanisms. In one retrospective cohort study including 79 hospitalized patients, COVID-19 encephalopathy did not cause great changes in CSF in terms of cell count and protein amount, but an increase in neuronal injury biomarker neuron-specific enolase (NSE) [34], suggesting a minor, if any, role for viral meningitis in neurologic symptoms.

In the majority of our patients, phenotypic characteristics were similar to those of primary tension-type headache (Table 3), but the presence of fever in most patients and a confirmed viral infection (COVID19) leads to a more accurate classification under "headache attributed to systemic viral infection" [12]. The most accurate clinical characteristic to allow this differentiation was the presence of fever. Headache characteristics in COVID-19 may change over the course of the disease $[9,13,37]$, possibly denoting a multifactorial role in the genesis of this symptom, including hypoxia-related headache and even headache secondary to personal protective equipment (PPE) use [11].

We have not found any particular characteristic that could help differentiate COVID-19-associated headache from headache secondary to other viral infections or noninfectious diseases, as was the case with some authors before us $[15,36]$. Nonetheless, we believe to have contributed to a more thorough description of its characteristics, which might allow together with other studies to increase awareness of physicians to headache as a common and sometimes initial symptom of COVID-19 infection.

This study has some limitations. This was a retrospective study based on review of clinical charts. Only charts of patients which reported neurological symptoms were reviewed, and, among these, only patients with headache were submitted to a specific interview. Thus, we were not able to determine overall headache prevalence in COVID19 patients or to compare data from patients with headache to those with COVID-19 and no neurological symptoms. There was a selection bias, as one of the health centers involved in the study is a reference center for stroke. Some patients were unable to fully inform about their symptoms due to severe systemic disease, encephalopathy, or aphasia, among other factors. The main strengths of the study were the fact that all patients had a laboratory-confirmed acute COVID-19 infection and that they were all submitted to a comprehensive, uniform interview for the characterization of headache.

\section{Conclusion}

Headache is a highly prevalent symptom in COVID-19. Its mechanisms are not completely understood, and its clinical characteristics are varied. Most cases are associated with fever and systemic inflammation. Characterization of headache is a challenge due to the fact that it is probably caused 
by multifactorial etiology and changes with the course of the disease.

Headache tends to begin in the first days of symptoms, be frontal or holocranial and last for days. A pressure quality and worsening with cough or physical activity is reported in most cases. It is associated with anosmia in a minority of patients. We have not found any characteristic that could differentiate COVID-19-associated headache from other causes of headache, possibly because of its multifactorial mechanism.

Further studies with larger samples, of a prospective nature with multiple points of clinical data collection and analysis of systemic and CSF inflammatory markers, could help clarify the mechanisms of headache in COVID-19 patients and its association with other neurological symptoms and severity of systemic disease.

Funding The authors (Pedro Braga Neto and Raquel Carvalho Montenegro) received funding from the Brazilian National Council for Scientific and Technological Development $(\mathrm{CNPq})$ and Fundação de Apoio ao Desenvolvimento Científico e Tecnológico (FUNCAP) as research grant funding. We also acknowledge that the authors (Pedro Braga Neto and Raquel Carvalho Montenegro) received the Brazilian funding numbers 88881.505364/2020-01 and 03195011/2020, respectively, from Coordenação de Aperfeiçoamento de Pessoal de Nível Superior - Brasil (CAPES) and FUNCAP and also acknowledge the support of Universidade Federal do Ceará (UFC), Hospital Geral de Fortaleza (HGF), and Hospital São José de Doenças Infecciosas (HSJ).

Data Availability All materials used in this study will be made available subject to a material transfer agreement.

Code Availability Not applicable.

\section{Declarations}

Ethics Approval The study was approved by the local ethics committee under the number 4092933.

Consent for Publication The manuscript contains no any individual person's data in any form.

Competing Interests The authors declare no competing interests.

\section{References}

1. https://www. who.int/emergencies/diseases/novel-coron avirus-2019

2. https://www.worldometers.info/coronavirus/

3. Mao L, Jin H, Wang M, Hu Y, Chen S, He Q et al (2020) Neurologic manifestations of hospitalized patients with coronavirus disease 2019 in Wuhan, China. JAMA Neurol 77:403-527. https:// doi.org/10.1001/jamaneurol.2020.1127

4. Armocida D, Palmieri M, Frati A, Santoro A, Pesce A (2020) How SARS-Cov-2 can involve the central nervous system. A systematic analysis of literature of the department of human neurosciences of Sapienza University, Italy. J Clin Neurosci 79:231-236. https:// doi.org/10.1016/j.jocn.2020.07.007

5. Niazkar HR, Zibaee B, Nasimi A, Bahri N (2020) The neurological manifestations of COVID-19: a review article. Neurol Sci 41(7):1667-1671. https://doi.org/10.1007/s10072-020-04486-3

6. Abboud H, Abboud FZ, Kharbouch H, Arkha Y, El Abbadi N, El Ouahabi A (2020) COVID-19 and SARS-Cov-2 infection: pathophysiology and clinical effects on the nervous system. World Neurosurg 140:49-53. https://doi.org/10.1016/j.wneu.2020.05.193

7. Islam MA, Alam SS, Kundu S, Hossan T, Kamal MA, Cavestro C (2020) Prevalence of headache in patients with coronavirus disease 2019 (COVID-19): a systematic review and meta-analysis of 14,275 patients. Front Neurol 11:562634. https://doi.org/10. 3389/fneur.2020.562634

8. Divani AA, Andalib S, Biller J, Di Napoli M, Moghimi N, Rubinos CA, O'Hana Nobleza C, Sylaja PN, Toledano M, Lattanzi S, McCullough LD, Cruz-Flores S, Torbey M, Azarpazhooh MR (2020) Correction to: Central nervous system manifestations associated with COVID-19. Curr Neurol Neurosci Rep 20(12):66. https://doi.org/10.1007/s11910-020-01086-8

9. Toptan T, Aktan Ç, Başarı A, Bolay H (2020) Case series of headache characteristics in COVID-19: headache can be an isolated symptom. Headache 60(8):1788-1792. https://doi.org/10.1111/ head. 13940

10. Trigo J, García-Azorín D, Planchuelo-Gómez Á, Martínez-Pías E, Talavera B, Hernández-Pérez I, Valle-Peñacoba G, Simón-Campo P, de Lera M, Chavarría-Miranda A, López-Sanz C, GutiérrezSánchez M, Martínez-Velasco E, Pedraza M, Sierra Á, GómezVicente B, Arenillas JF, Guerrero ÁL (2020) Factors associated with the presence of headache in hospitalized COVID-19 patients and impact on prognosis: a retrospective cohort study. J Headache Pain 21(1):94. https://doi.org/10.1186/s10194-020-01165-8

11. Porta-Etessam J, Matías-Guiu JA, González-García N, Gómez Iglesias P, Santos-Bueso E, Arriola-Villalobos P, García-Azorín D, Matías-Guiu J (2020) Spectrum of headaches associated with SARS-CoV-2 infection: study of healthcare professionals. Headache 60(8):1697-1704. https://doi.org/10.1111/head.13902

12. Headache Classification Committee of the International Headache Society (IHS) (2018) The International Classification of Headache Disorders, 3rd edition. Cephalalgia 38(1):1-211. https://doi.org/ 10.1177/0333102417738202

13. Xu XW, Wu XX, Jiang XG, Xu KJ, Ying LJ, Ma CL, Li SB, Wang HY, Zhang S, Gao HN, Sheng JF, Cai HL, Qiu YQ, Li LJ (2020) Clinical findings in a group of patients infected with the 2019 novel coronavirus (SARS-Cov-2) outside of Wuhan, China: retrospective case series. BMJ. https://doi.org/10.1136/bmj.m606

14. Suzuki K (2020) Coronavirus disease 2019 (COVID-19) and headaches. Rinsho Shinkeigaku 60(9):589-596. https://doi.org/ 10.5692/clinicalneurol.cn-001490

15. López JT, García-Azorín D, Planchuelo-Gómez Á, García-Iglesias C, Dueñas-Gutiérrez C, Guerrero ÁL (2020) Phenotypic characterization of acute headache attributed to SARS-CoV-2: an ICHD-3 validation study on 106 hospitalized patients. Cephalalgia 40(13):1432-1442. https://doi.org/10.1177/0333102420 965146

16. Naqvi AAT, Fatima K, Mohammad $T$ et al (2020) Insights into SARS-CoV-2 genome, structure, evolution, pathogenesis and therapies: structural genomics approach. Biochim Biophys Acta Mol Basis Dis 1866(10):165878. https://doi.org/10.1016/j.bbadis. 2020.165878

17. Bolay H, Gül A, Baykan B (2020) COVID-19 is a Real Headache! Headache. https://doi.org/10.1111/head.13856

18. Nisticò R, Salter E, Nicolas C, Feligioni M, Mango D, Bortolotto ZA, Gressens P, Collingridge GL, Peineau S (2017) Synaptoimmunology - roles in health and disease. Mol Brain 10(1):26. https://doi.org/10.1186/s13041-017-0308-9 
19. Liu W, Tang Y, Feng J (2011) Cross talk between activation of microglia and astrocytes in pathological conditions in the central nervous system. Life Sci 89(5-6):141-146. https://doi.org/10. 1016/j.lfs.2011.05.011

20. Liu Y, Yang Y, Zhang C, Huang F, Wang F, Yuan J, Wang Z, Li J, Li J, Feng C, Zhang Z, Wang L, Peng L, Chen L, Qin Y, Zhao D, Tan S, Yin L, Xu J, Zhou C, ... Liu L (2020) Clinical and biochemical indexes from 2019-nCoV infected patients linked to viral loads and lung injury. Sci China Life Sci 63(3):364-374. https://doi.org/10.1007/s11427-020-1643-8

21. Chen L, Wang G, Long X, Hou H, Wei J, Cao Y, Tan J, Liu W, Huang L, Meng F, Huang L, Wang N, Zhao J, Huang G, Sun Z, Wang W, Zhou J (2021) Dynamics of blood viral load is strongly associated with clinical outcomes in coronavirus disease 2019 (COVID-19) patients: a prospective cohort study. J Mol Diagn 23(1):10-18. https://doi.org/10.1016/j.jmoldx.2020.10.007

22. Chen W, Lan Y, Yuan X, Deng X, Li Y, Cai X, Li L, He R, Tan Y, Deng X, Gao M, Tang G, Zhao L, Wang J, Fan Q, Wen C, Tong Y, Tang Y, Hu F, Li F, ... Tang X (2020) Detectable 2019-nCoV viral RNA in blood is a strong indicator for the further clinical severity. Emerg Microbes Infect 9(1):469-473. https://doi.org/10. 1080/22221751.2020.1732837

23. Varga Z, Flammer AJ, Steiger P, Haberecker M, Andermatt R, Zinkernagel AS, Mehra MR, Schuepbach RA, Ruschitzka F, Moch H (2020) Endothelial cell infection and endotheliitis in COVID-19. Lancet 395(10234):1417-1418. https://doi.org/10. 1016/S0140-6736(20)30937-5

24. Patil J, Schwab A, Nussberger J, Schaffner T, Saavedra JM, Imboden H (2010) Intraneuronal angiotensinergic system in rat and human dorsal root ganglia. Regul Pept 162(1-3):90-98. https://doi.org/10.1016/j.regpep.2010.03.004

25. Desforges M, Le Coupanec A, Dubeau P, Bourgouin A, Lajoie L, Dubé M, Talbot PJ (2019) Human coronaviruses and other respiratory viruses: underestimated opportunistic pathogens of the central nervous system? Viruses 12(1):14. https://doi.org/10. 3390/v12010014

26 Barón-Sánchez J, Santiago C, Goizueta-San Martín G, Arca R, Fernández R (2020) Smell and taste disorders in Spanish patients with mild COVID-19. Neurologia. 35(9):633-638. https://doi.org/ 10.1016/j.nrl.2020.07.006 (English, Spanish)

27. Hou CC, Lin H, Chang CP, Huang WT, Lin MT (2011) Oxidative stress and pyrogenic fever pathogenesis. Eur J Pharmacol 667(1-3):6-12. https://doi.org/10.1016/j.ejphar.2011.05.075
28. Zhao Z, Wei Y, Tao C (2021) An enlightening role for cytokine storm in coronavirus infection. Clin Immunol 222:108615. https:// doi.org/10.1016/j.clim.2020.108615

29. Lukacs M, Tajti J, Fulop F, Toldi J, Edvinsson L, Vecsei L (2017) Migraine, neurogenic inflammation, drug development - pharmacochemical aspects. Curr Med Chem 24(33):3649-3665. https:// doi.org/10.2174/0929867324666170712163437

30. Xanthos DN, Sandkühler J (2014) Neurogenic neuroinflammation: inflammatory CNS reactions in response to neuronal activity. Nat Rev Neurosci 15(1):43-53. https://doi.org/10.1038/nrn3617

31. Moskowitz MA, Reinhard JF Jr, Romero J, Melamed E, Pettibone DJ (1979) Neurotransmitters and the fifth cranial nerve: is there a relation to the headache phase of migraine? Lancet (London, England) 2(8148):883-885. https://doi.org/10.1016/s0140-6736(79) 92692-8

32. Buture A, Gooriah R, Nimeri R, Ahmed F (2016) Current understanding on pain mechanism in migraine and cluster headache. Anesth Pain Med 6(3):e35190. https://doi.org/10.5812/aapm. 35190

33. Ye M, Ren Y, Lv T (2020) Encephalitis as a clinical manifestation of COVID-19. Brain Behav Immun 88:945-946. https://doi.org/ 10.1016/j.bbi.2020.04.017

34. Toklu H, Ganti L, Crimi E, Cintron C, Hagan J, Serrano E (2020) Cerebrospinal fluid findings and hypernatremia in COVID-19 patients with altered mental status. Int J Emerg Med 13(1):63. https://doi.org/10.1186/s12245-020-00327-4

35. Tsivgoulis G, Palaiodimou L, Zand R, Lioutas VA, Krogias C, Katsanos AH, Shoamanesh A, Sharma VK, Shahjouei S, Baracchini C, Vlachopoulos C, Gournellis R, Sfikakis PP, Sandset EC, Alexandrov AV, Tsiodras S (2020) COVID-19 and cerebrovascular diseases: a comprehensive overview. Ther Adv Neurol Disord 13:1756286420978004. https://doi.org/10.1177/1756286420 978004

36. Silva MTT, Lima MA, Torezani G, Soares CN, Dantas C, Brandão CO, Espíndola O, Siqueira MM, Araujo AQ (2020) Isolated intracranial hypertension associated with COVID-19. Cephalalgia 40(13):1452-1458. https://doi.org/10.1177/0333102420965963

37. Belvis R (2020) Headaches during COVID-19: my clinical case and review of the literature. Headache 60(7):1422-1426. https:// doi.org/10.1111/head.13841

Publisher's Note Springer Nature remains neutral with regard to jurisdictional claims in published maps and institutional affiliations. 